Pathologe 2012 · [Suppl 2] 33:347-347

DOI 10.1007/s00292-012-1657-x

Online publiziert: 13. September 2012

๑) Springer-Verlag 2012

\author{
A. Burkhardt \\ Pathologiepraxis Reutlingen
}

\section{Bericht der AG Oralpathologie}

141 Proben durch Dreyer et al. Sie bestätigen, dass der p-16-Nachweis ein geeigneter Surrogatmarker einer HPV-Assoziation an oralen und oropharyngealen Karzinomen in der Routinediagnostik darstellt. Diese Untersuchung ist wichtig, weil diese Karzinome eine bessere Prognose aufweisen, dabei ist sie prädiktiv für das Ansprechen auf eine Radiotherapie, unabhängig von einer begleitenden Anti-EGFR- oder Chemotherapie. Eine zusätzliche EGFRInhibition erzielt dabei deutlich höhere Überlebensraten (Untersuchung an 73 Patienten, Stenziger et al.).

EBV-assoziierte lymphoproliferative Erkrankungen nach Transplantation (PTLD, „post-transplant lymphoproliferative disorder") manifestieren sich hauptsächlich an den Tonsillen, hier meist als Frühläsion oder polymorphe PTLD, während die monomorphe Form hier selten ist (Tiede et al.).

Weitere Präsentationen beschäftigten sich mit verschiedenen karzinomassoziierten Faktoren:

- SOX2-Amplifikation als Hinweis auf eine Beziehung von sinonasalen undifferenzierten Karzinomen (SNUC) und undifferenzierten Plattenepithelkarzinomen (Göke et al.) und auf ein malignes Potenzial in Vorläuferläsionen, z. B. invertierten Papillomen;

- Caspase14 als Differenzierungsfaktor in Leukoplakie und Oralkarzinom (Scharenberg et al.);

- FGFR1-Amplifikation, die eine HPV-Assoziation ausschließt, könnte durch entsprechende Inhibitoren bei inoperablen Karzinomen therapeutisch von Bedeutung sein (Göke et al.);

- EGFR-Inhibitor-Sensitivität von Karzinomen, die durch stromale Fibroblasten, die unter Umständen als Myofibroblasten akkumulieren, modifiziert wird (Richter et al.).

Die Studie von Weiler et al. beschäftigte sich mit nichtsebazischen Adenomen der Speicheldrüsen anhand von 9 Fällen. Sie vermuten, dass sie sich aus embryonalen Speicheldrüseneinschlüssen in intra-/periparotidalen Lymphknoten entwickeln. Ihre Kenntnis ist differenzialdiagnostisch wichtig, um Fehldiagnosen - z. B. lymphoepitheliale Karzinome - zu vermeiden (in $20 \%$ lagen primäre Fehldiagnosen vor.) Wichtig für die korrekte Einschätzung ist hier die Darstellung der Proliferationsrate (Ki67).

Eine Serie von 23 seltenen lipomatösen Speicheldrüsentumoren (ohne pleomorphe Adenome oder Myoepitheliome) wurde von Agaimy et al. demonstriert. Neben gewöhnlichen Lipomen handelt es sich um onkozytische Lipomadenome und nichtonkozytische Sialolipome. Bei der letztgenannten findet sich auch eine sebazische Differenzierung. Ein Risiko einer malignen Transformation besteht offenbar nicht.

Ein einschlägig dokumentiertes Angiosarkom der Tonsille (Agaimy et al.) wurde als diagnostische Fallgrube präsentiert.

\section{Korrespondenzadresse}

Prof. Dr. A. Burkhardt

Pathologiepraxis Reutlingen

Obere Wässere 3-7, 72764 Reutlingen

pathologiepraxis@arcor.de

Interessenkonflikt. Der korrespondierende Autor gibt an, dass kein Interessenkonflikt besteht. The supplement this article is part of is not sponsored by the industry.
Die Diagnostik der HPV-Infektion am Gewebe war Gegenstand einer Studie an 